\title{
Proceso para fortalecer el registro de propiedad intelectual en las instituciones de educación superior*
}

[Notas metodológicas y de investigación]

\author{
Denisse Patricia Ruiz Pintor** \\ Marisol Morillo Ubaque ${ }^{* * *}$ \\ Paula Peralta Pérez ${ }^{* * * *}$
}

Recibido: 1 de septiembre de 2020

Revisado: 10 de septiembre de 2020

Aceptado: 11 de septiembre de 2020

\footnotetext{
* Nota metodológica

** Economista. Especialista en Psicología del Consumidor. Instructora en el Servicio Nacional de Aprendizaje (SENA). Bogotá, Colombia. Correo electrónico: pruizp@misena.edu.co. ORCID: https://orcid.org/0000-0001-5870-5319. CvLAC: https://scienti.minciencias.gov.co/cvlac/visualizador/generarCurriculoCv.do?cod_rh $=0$ 001509677

${ }_{* * *}$ Estudiante de pregrado en Administración Financiera de la Universidad Minuto de Dios. Bogotá, Colombia. Correo electrónico: mmorillouba@uniminuto.edu.co. ORCID: https://orcid.org/0000-0001-6383-1731. CvLAC:

https://scienti.minciencias.gov.co/cvlac/visualizador/generarCurriculoCv.do?cod_rh $=0$ 000165286

**** Estudiante del programa de Tecnólogo en Gestión Bancaria y Financiera del Servicio Nacional de Aprendizaje (SENA). Bogotá, Colombia. Correo electrónico: pperalta4@misena.edu.co. ORCID: https://orcid.org/0000-0003-3230-7982. CvLAC: https://scienti.minciencias.gov.co/cvlac/visualizador/generarCurriculoCv.do?cod_rh $=0$ 001762046
} 
Cómo citar este artículo:

Ruiz Pintor, D. P., Morillo Ubaque, M. y Peralta Pérez, P. (2020). Proceso para fortalecer el registro de propiedad intelectual en las instituciones de educación superior. Signos, Investigación en sistemas de gestión, 13(1).

https://doi.org/10.15332/24631140.6339

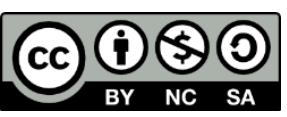

\section{Resumen}

Esta nota metodológica propone un documento de referencia para el registro de proyectos desarrollados en las instituciones de educación superior (IES), en el que de forma breve se define un problema, se realiza una síntesis de la evidencia, se identifican posibles líneas de actuación y se proponen recomendaciones. El objetivo principal es describir la metodología para la valoración y el registro de la producción intelectual de estudiantes de IES en diez etapas: 1) fortalecimiento del conocimiento sobre registro de propiedad intelectual en los contenidos curriculares; 2) desarrollo de proyectos de emprendimiento o investigación en los ambientes de formación; 3) implementación de base de datos para registro de proyectos e innovaciones; 4) registro oficial de los proyectos en la base de datos; 5) revisión y evaluación de proyectos por parte de los expertos en propiedad intelectual de la institución; 6) recomendaciones para los proyectos que requieran ajustes; 7) consideración de alternativas en las categorías de registro de propiedad intelectual de los proyectos aprobados; 8) registro de propiedad intelectual en las instituciones responsables (Superintendencia de Industria y Comercio, Dirección Nacional de Derechos de Autor); 9) implementación de los proyectos que lo ameriten; 10) divulgación de los proyectos en las diferentes instituciones educativas. Por último, se presenta el proceso de registro de la propiedad intelectual como material de indagación para la gestión de cualquier tipo de proyectos.

Palabras clave: proyectos, proceso de registro, propiedad intelectual, registro de marca, derechos de autor, repositorio. 


\section{Process to strengthen intellectual property registration in higher education institutions}

\section{Abstract}

This methodological note proposes a reference document for the registration of projects developed in higher education institutions (HEI), in which a problem is briefly defined, a synthesis of the evidence is made, possible lines of action are identified, and recommendations are proposed. The main objective is to describe the methodology for the assessment and registration of the intellectual production of students of HEI in ten stages: 1) strengthening knowledge on intellectual property registration in the curricular contents; 2) development of entrepreneurial or research projects in the training environments; 3) implementation of database for projects and innovations registration; 4) official registration of projects in the database; 5 ) review and evaluation of projects by the institution's intellectual property experts; 6) recommendations for projects requiring adjustments; 7) inclusion of alternatives in the intellectual property registration categories of approved projects; 8) registration of intellectual property in the responsible institutions (Superintendence of Industry and Commerce, Copyright National Directorate); 9) implementation of the projects requiring implementation; 10) dissemination of projects in the different educational institutions. Finally, the process of intellectual property registration is presented as research material for the any type of project management.

Keywords: projects, registration process, intellectual property, trademark registration, copyright, repository. 


\section{Processo para o fortalecimento do registro de propriedade intelectual nas instituições de ensino superior}

\section{Resumo}

Esta nota metodológica propõe um documento de referência para o registro de projetos desenvolvidos em instituições de ensino superior (IES), no qual se define um problema de forma breve, realiza-se uma síntese das evidências, identificam-se as possíveis linhas de ação e, em consequência, são propostas as recomendações. O principal objetivo é descrever a metodologia para a avaliação e registro da produção intelectual dos estudantes de IES em dez estágios: 1) fortalecimento do conhecimento sobre registro de propriedade intelectual nos conteúdos curriculares; 2) desenvolvimento de projetos de empreendedorismo ou pesquisa nos ambientes de treinamento; 3) implementação de banco de dados para registro de projetos e inovações; 4) registro oficial de projetos no banco de dados; 5) revisão e avaliação de projetos pelos especialistas em propriedade intelectual da instituição; 6) recomendações para projetos que requerem ajustes; 7) consideração de alternativas nas categorias de registro de propriedade intelectual de projetos aprovados; 8) registro de propriedade intelectual nas instituições responsáveis (Superintendência de Indústria e Comércio, Diretoria Nacional de Direitos Autorais); 9) implementação dos projetos que o mereçam; 10) divulgação de projetos nas diferentes instituições de ensino. Por fim, o processo de registro de propriedade intelectual é apresentado como material de pesquisa para a gestão de qualquer tipo de projeto.

Palavras-chave: projetos, processo de registro, propriedade intelectual, registro de marcas, direitos autorais, repositório.

\section{Introducción}

Los procesos de registro de la propiedad intelectual en las instituciones de educación superior son herramientas para la gestión de la innovación y el 
conocimiento en estas entidades. La presente nota metodológica aborda el tema de la propiedad intelectual, definida por la Organización Mundial de la Propiedad Intelectual (OMPI, s. f.) como

[...] las creaciones de la mente: invenciones, obras literarias y artísticas, así como símbolos, nombres e imágenes utilizados en el comercio. Los derechos de la propiedad intelectual se asemejan a cualquier otro derecho de propiedad, ya que permite al creador, o al titular de una patente, marca o derecho de autor, gozar de los beneficios que derivan de su obra o de la inversión realizada en relación con una creación.

Esos derechos están consagrados en el artículo 27 de la Declaración Universal de los Derechos Humanos (Naciones Unidas, 1948), que contempla el derecho a beneficiarse de la protección de los intereses morales y materiales resultantes de la autoría de las producciones científicas, literarias o artísticas. A continuación, se abordan los diferentes tipos y conceptos de propiedad intelectual como una rama del derecho que busca, por una parte, fomentar la innovación, creación y transferencia tecnológica y, por la otra, ordenar los mercados para facilitar la toma de decisiones por parte del público consumidor (Instituto Nacional de Propiedad Industrial [Inapi], s. f.).

La propiedad intelectual se define en dos categorías. Por un lado, se encuentran los derechos de autor, que abarcan obras literarias (novelas, poemas, obras de teatro), obras audiovisuales (películas y música) y obras artísticas (dibujos, pinturas, fotografías, esculturas y diseños arquitectónicos) que están sujetas a protección (Centro Colombiano del Derecho de Autor [Cecolda], s. f.). Por otro lado, está la propiedad industrial, que abarca las patentes de invención, las marcas, los diseños industriales y las indicaciones geográficas. Con relación a la propiedad industrial, las patentes son un derecho exclusivo concedido sobre una 
invención, producto o proceso que constituye una nueva manera de hacer algo o propone una nueva solución técnica a un problema (OMPI, 2011).

En ese sentido, las patentes constituyen un reconocimiento a la creatividad, brindan la posibilidad de obtener una recompensa material por las creaciones comercializables y alientan la innovación que, a su vez, mejora la calidad de vida (OMPI, 2011). En este contexto, los modelos de utilidad son derechos de exclusividad de uso y explotación durante un periodo de tiempo limitado que se le da al titular de una invención de bajo valor creativo o innovación. El diseño industrial constituye el aspecto ornamental o estético de un artículo y, por último, las marcas permiten diferenciar los productos o servicios de una empresa de los de otra (Inapi, s. f.)

Pedreros (2017) considera que la propiedad industrial y los derechos de autor hacen parte del derecho de la propiedad intelectual:

Esta es una de las formas en las que las que la ciencia jurídica positiva protege el dominio que una persona posee naturalmente sobre su actividad creativa en cuanto bien inmaterial o incorporal susceptible de explotación económica y que el mismo tiene dos componentes: i) el intelectual que trata los derechos de autor y ii) el económico que conlleva la propiedad industrial. (Carreras, 2008, como se citó en Pedreros, 2017, p. 6)

En términos generales, la propiedad industrial "protege jurídicamente las invenciones, marcas de fábrica o comercio, los dibujos o modelos industriales, el nombre comercial, las denominaciones de origen e indicaciones de procedencia, y reprime los actos de competencia desleal que se pueden presentar en el tráfico mercantil" (García-Huidobro, 1992, como se citó en Pedreros, 2017, p. 6). 
En el marco de la teoría económica, los derechos de propiedad se pueden clasificar, según Cristancho (2017), en iusnaturalistas y utilitaristas. La postura iusnaturalista, que corresponde al siglo XIX, sostiene que "los individuos son los dueños de su cuerpo y trabajo, por tanto, también de sus frutos, y dichas creaciones serían esos frutos" (p. 128). Los derechos de propiedad intelectual son connaturales a las creaciones humanas, ya que quien invierte su tiempo, trabajo y talento debe obtener una retribución a cambio, la cual debe ser respetada por el ordenamiento jurídico (Cristancho, 2017).

Esta situación se presenta actualmente en diversidad de productos, servicios, proyectos e investigaciones. Como menciona Cristancho (2017), los denominados free riders copian las ideas de los creadores de proyectos y las venden a un menor costo, puesto que no tuvieron que asumir ninguna carga financiera durante el proceso investigativo. Por lo anterior, es importante otorgar derechos exclusivos de explotación a los productores de conocimiento para que puedan recuperar sus costos fijos y se motiven a producir.

De igual forma, Olarte Reyes y Lis-Gutiérrez (2015) definen el capital intelectual como material intelectual, conocimiento, información, propiedad intelectual y experiencia, que puede utilizarse para crear valor en las organizaciones.

Actualmente, la mayoría de los proyectos de innovación o documentos de investigación generados por estudiantes y profesores quedan en el papel o en un computador, y no son postulados a registro de derechos de autor, patentes, marca o diseño industrial. Si se registrara esta gran capacidad que tienen las instituciones educativas en el desarrollo de proyectos, podría generar un alto volumen de producción intelectual. 
Para solucionar esta problemática, se propone un manual de proceso para el registro de proyectos en el que, inicialmente, se plasman los pasos a seguir para fomentar el registro de propiedad intelectual en las universidades. El objetivo de este manual es divulgar, motivar y fortalecer en la comunidad académica la importancia del registro de la propiedad intelectual en cualquiera de sus categorías. Este instrumento de comunicación busca que en un futuro cercano las instituciones de educación superior de Colombia se destaquen en Latinoamérica por su nivel de registro de propiedad intelectual.

\section{Procedimiento para el registro de propiedad intelectual}

Paso 1. Fortalecimiento del conocimiento sobre registro de propiedad intelectual en los contenidos curriculares

Puede hacerse a través de un módulo donde se enseñe el proceso de registro de propiedad intelectual, las entidades responsables, los costos, etc.

Paso 2. Desarrollo de proyectos de emprendimiento o investigación en los ambientes de formación

En la mayoría de instituciones académicas se imparten materias relacionadas con el desarrollo de proyectos empresariales, innovaciones o mejoras en productos o servicios y trabajos de investigación. Con el apoyo del departamento de propiedad intelectual de la universidad, estos productos pueden postularse para el registro de propiedad intelectual según la modalidad de mayor afinidad. 
Paso 3. Implementación de base de datos para registro de proyectos e innovaciones

Es importante desarrollar e implementar una base de datos para el registro de los proyectos que permita organizarlos de manera adecuada y proteger los derechos de autor de sus creadores.

Paso 4. Registro oficial de los proyectos en base de datos

El registro de los proyectos se debe realizar de forma ordenada y sistemática con una estructura general que facilite su evaluación. Se deben clasificar según la posible categoría a la que pertenecen: registro de marca, derechos de autor, patente, etc.

Paso 5. Revisión y evaluación de proyectos por parte de los expertos en propiedad intelectual de la institución

El proyecto, innovación o marca ingresará a un proceso de revisión en el que los encargados del procedimiento validarán que estos cumplan con los requisitos de acuerdo con su categoría de registro (registro de marca, patente, diseño industrial, derechos de autor, indicaciones geográficas). Darán su visto bueno para el registro o indicarán si el trabajo requiere ajustes.

\section{Paso 6. Recomendaciones para los proyectos que requieran ajustes}

Si los proyectos requieren ajustes, les serán enviadas las recomendaciones puntuales en cada uno de los aspectos a mejorar. 


\section{Paso 7. Consideración de alternativas para categorías de registro de los proyectos aprobados}

En esta etapa se debe verificar la clasificación realizada en el registro para validar la categoría teniendo en cuenta la tabla 1 y realizar los ajustes necesarios. Por ejemplo, para un proyecto empresarial, se podría generar el registro de marca y/o patente de acuerdo con su naturaleza, o para un proyecto de investigación que resulte en un artículo, se puede aplicar la protección de derechos de autor.

Tabla 1. Categorías de propiedad intelectual

\begin{tabular}{|c|c|c|c|}
\hline \multicolumn{2}{|c|}{ Categoría } & Definición & $\begin{array}{l}\text { Plazo de vigencia } \\
\text { de la protección }\end{array}$ \\
\hline \multicolumn{2}{|l|}{ Marca } & $\begin{array}{l}\text { Signo visible y novedoso que identifica a } \\
\text { productos, servicios o empresas, } \\
\text { incluidos los sonidos y los atributos } \\
\text { identificables por el olfato. }\end{array}$ & 10 años renovables \\
\hline \multicolumn{2}{|c|}{ Indicación geográfica (IG) } & $\begin{array}{l}\text { Nombre que identifica a los productos } \\
\text { como originarios de un territorio } \\
\text { específico, cuando su calidad se atribuye } \\
\text { fundamentalmente al origen geográfico. } \\
\text { Varios productores pueden usar una } \\
\text { misma indicación. }\end{array}$ & A perpetuidad \\
\hline \multicolumn{2}{|c|}{ Nombre del dominio } & $\begin{array}{l}\text { Nombre exclusivo acompañado de un } \\
\text { número del protocolo de internet que } \\
\text { identifica a los computadores en la red. } \\
\text { Registrado por la corporación de internet } \\
\text { para la asignación de nombres y } \\
\text { números. }\end{array}$ & 3 años renovables \\
\hline \multirow[t]{4}{*}{$\begin{array}{l}\text { Patentes } \\
\text { y otros }\end{array}$} & $\begin{array}{l}\text { Patente de } \\
\text { invención }\end{array}$ & $\begin{array}{l}\text { Innovación que muestre un nivel } \\
\text { inventivo suficiente y tenga aplicación } \\
\text { industrial. }\end{array}$ & $\begin{array}{l}20 \text { años no } \\
\text { renovables }\end{array}$ \\
\hline & $\begin{array}{l}\text { Modelo de } \\
\text { utilidad }\end{array}$ & $\begin{array}{l}\text { Artículo cuya forma sea reivindicable, } \\
\text { tanto en su aspecto externo como en su } \\
\text { funcionamiento, y preste alguna utilidad. }\end{array}$ & $\begin{array}{l}10 \text { años no } \\
\text { renovables }\end{array}$ \\
\hline & $\begin{array}{l}\text { Diseño } \\
\text { industrial }\end{array}$ & $\begin{array}{l}\text { Toda forma que sirva de patrón para } \\
\text { fabricar otras unidades, siempre que sea } \\
\text { novedosa y tenga una apariencia especial } \\
\text { perceptible. }\end{array}$ & $\begin{array}{l}10 \text { años no } \\
\text { renovables }\end{array}$ \\
\hline & $\begin{array}{l}\text { Información no } \\
\text { divulgada }\end{array}$ & $\begin{array}{l}\text { Datos de pruebas para obtener } \\
\text { autorización de las autoridades } \\
\text { competentes a fin de comercializar un } \\
\text { producto determinado. Relacionados con } \\
\text { la eficacia y seguridad de los productos } \\
\text { farmacéuticos y químico-agrícolas cuya }\end{array}$ & $\begin{array}{l}5^{-10} \text { años no } \\
\text { renovables }\end{array}$ \\
\hline
\end{tabular}




\begin{tabular}{|c|c|c|c|}
\hline \multicolumn{2}{|c|}{ Categoría } & Definición & $\begin{array}{l}\text { Plazo de vigencia } \\
\text { de la protección }\end{array}$ \\
\hline \multirow{2}{*}{\multicolumn{2}{|c|}{ Variedad vegetal }} & $\begin{array}{l}\text { creación exigió un esfuerzo considerable. } \\
\text { No pueden darse a conocer ni usarse } \\
\text { para aprobar productos genéricos. }\end{array}$ & \\
\hline & & $\begin{array}{l}\text { Conjunto de plantas comercialmente } \\
\text { nuevas, distintas, homogéneas y estables } \\
\text { de un solo taxón botánico del rango más } \\
\text { bajo conocido, que pueda definirse por la } \\
\text { expresión de los caracteres resultantes de } \\
\text { uno o varios genotipos. }\end{array}$ & $\begin{array}{l}20-25 \text { años no } \\
\text { renovables }\end{array}$ \\
\hline \multirow[t]{2}{*}{$\begin{array}{l}\text { Derechos } \\
\text { de autor } \\
\text { y } \\
\text { conexos }\end{array}$} & $\begin{array}{l}\text { Derechos de } \\
\text { autor }\end{array}$ & $\begin{array}{l}\text { Varios derechos exclusivos tales como los } \\
\text { de reproducción, distribución y otros } \\
\text { respecto de formas de expresión. } \\
\text { Incluyen el software y las compilaciones } \\
\text { de datos, pero no los datos propiamente } \\
\text { dichos. No protegen ideas, } \\
\text { procedimientos, métodos de operación o } \\
\text { conceptos matemáticos. }\end{array}$ & \multirow[t]{2}{*}{$\begin{array}{l}\text { Según el Adpic, } 50 \\
\text { años a contar desde } \\
\text { la muerte del autor. } \\
\text { Según los TLC, } 70 \\
\text { años no renovables. }\end{array}$} \\
\hline & $\begin{array}{l}\text { Derechos } \\
\text { conexos }\end{array}$ & $\begin{array}{l}\text { Varios derechos exclusivos de artistas } \\
\text { intérpretes, ejecutantes y productores de } \\
\text { fonogramas respecto de sus } \\
\text { interpretaciones, ejecuciones o } \\
\text { fonogramas (reproducción, } \\
\text { radiodifusión, comunicación al público y } \\
\text { otros). }\end{array}$ & \\
\hline \multirow[t]{3}{*}{$\begin{array}{l}\text { Otras } \\
\text { formas } \\
\text { de } \\
\text { control }\end{array}$} & $\begin{array}{l}\text { Secreto } \\
\text { comercial }\end{array}$ & $\begin{array}{l}\text { Información confidencial bajo control } \\
\text { privado, no fácilmente accesible para } \\
\text { expertos, que tenga valor comercial por } \\
\text { ser secreta y que haya sido objeto de } \\
\text { medidas razonables para mantenerla en } \\
\text { reserva. }\end{array}$ & $\begin{array}{l}\text { Protección legal } \\
\text { indefinida }\end{array}$ \\
\hline & $\begin{array}{l}\text { Medidas } \\
\text { tecnológicas de } \\
\text { protección }\end{array}$ & $\begin{array}{l}\text { Dispositivos, software o ambos que } \\
\text { controlan el acceso a contenidos e } \\
\text { informaciones a fin de mantenerlos en } \\
\text { reserva o para permitir solo un acceso } \\
\text { selectivo a ellos. }\end{array}$ & $\begin{array}{l}\text { Protección legal } \\
\text { indefinida }\end{array}$ \\
\hline & $\begin{array}{l}\text { Contratos } \\
\text { privados }\end{array}$ & $\begin{array}{l}\text { Acuerdo privado entre partes que puede } \\
\text { incluir licencias, arrendamientos u otras } \\
\text { formas contractuales que generan } \\
\text { derechos y obligaciones. Su alcance } \\
\text { puede ser mayor que el de las normas de } \\
\text { propiedad intelectual. }\end{array}$ & $\begin{array}{l}\text { Protección legal } \\
\text { según vigencia del } \\
\text { contrato }\end{array}$ \\
\hline
\end{tabular}

Adpic: Acuerdo sobre los Aspectos de los Derechos de Propiedad Intelectual relacionados con el Comercio de la Organización Mundial del Comercio. TLC: Tratado de Libre Comercio.

Fuente: adaptada de Díaz (2008). 
Paso 8. Registro ante las instituciones responsables

Después de haber cumplido con todos los requerimientos y evaluaciones, el proyecto está listo para ser registrado ante las entidades competentes, como la Superintendencia de Industria y Comercio o la Dirección Nacional de Derechos de Autor. Con el apoyo de su tutor de propiedad intelectual, los autores o generadores de los proyectos realizan el proceso de registro en línea o de manera presencial. Dependiendo de la categoría de registro, cada entidad tiene unos tiempos de respuesta diferentes.

\section{Paso 9. Implementación de los proyectos que lo ameriten}

Los proyectos productivos dirigidos al desarrollo de emprendimientos o pymes pueden ser asesorados para buscar financiación, ya sea en bancas de segundo piso, directamente bancos, cooperativas o fondos que tengan líneas de crédito con capital semilla para estas empresas.

\section{Paso 10. Divulgación de los proyectos en las diferentes instituciones educativas}

Finalmente, es importante socializar los proyectos exitosos que han logrado su registro de propiedad intelectual, para mostrar los beneficios de este tipo de procesos y motivar a la comunidad académica a implementarlos.

\section{Validación del procedimiento para el registro de marca}

Un entregable del proceso de registro de propiedad intelectual puede ser implementado en subsiguientes versiones para mejorar los procedimientos a nivel metodológico y tecnológico. De esta forma, se pueden agilizar los procesos en las instituciones e impactar positivamente las cifras sobre propiedad intelectual en instituciones académicas a nivel nacional. 


\section{Consideraciones finales}

El proceso de registro de propiedad intelectual de proyectos desarrollados en instituciones de educación superior se puede realizar de forma eficaz siguiendo los diez pasos aquí expuestos. La difusión de esta propuesta puede concientizar sobre la importancia de proteger los proyectos productivos, formativos y de investigación de los estudiantes en las instituciones de educación superior. Esto les permitirá recibir retribuciones educativas, económicas o laborales que los motiven a continuar desarrollando innovaciones. Asimismo, estas creaciones intelectuales pueden materializarse en objetos tangibles que contribuyan al desarrollo social y económico del país.

\section{Referencias}

Centro Colombiano del Derecho de Autor. (s. f.). Derecho de autor: preguntas frecuentes. http://www.cecolda.org.co/index.php/derecho-de-autor/preguntas-frecuentes

Cristancho, F. (2017). La propiedad intelectual en los acuerdos ADPIC plus suscritos por Colombia: una visión desde la teoría económica de los derechos de propiedad. CES Derecho, 8(1), 124-138. https://doi.org/10.21615/4274

Díaz, A. (2008). América Latina y el Caribe. La propiedad intelectual después de los tratados de libre comercio. Comisión Económica para América Latina y el Caribe.

Instituto Nacional de Propiedad Industrial. (s. f.). Conceptos fundamentales sobre la propiedad intelectual. Consultado el 15 de agosto de 2020. https://www.inapi.cl/portal/institucional/600/w3-article-1192.html

Naciones Unidas. (1948). Declaración Universal de los Derechos Humanos. https://www.ohchr.org/EN/UDHR/Documents/UDHR Translations/spn.pdf

Olarte Reyes, M., \& Lis-Gutiérrez, J. (2015). Gestión de activos intelectuales y creación de valor. Administración y Desarrollo, 45(2), 190-207. http://esapvirtual.esap.edu.co/ojs/index.php/admindesarro/article/view/11 
Organización Mundial de la Propiedad Intelectual. (s. f.). ¿Qué es la propiedad intelectual? Consultado el 15 de agosto de 2020. https://www.wipo.int/aboutip/es/

Organización Mundial de la Propiedad Intelectual. (2011). ¿Qué es una patente? En ¿Qué es la propiedad intelectual? (pp. 4-7).

https://www.wipo.int/edocs/pubdocs/es/intproperty/450/wipo_pub_450

Pedreros, H. N. (2017). Propiedad industrial en Colombia: los retos en la sociedad del conocimiento [Trabajo de pregrado, Universidad Católica de Colombia]. Repositorio Institucional Universidad Católica de Colombia. https://repository.ucatolica.edu.co/handle/10983/14297 